\title{
La enseñanza de la atenuación en E/LE a partir del análisis de un corpus real
}

DANIEL SECCHI

Université Picardie Jules Verne

Resumen: El presente trabajo busca evidenciar la potencialidad de los corpus discursivos orales reales como herramienta para la enseñanza/aprendizaje de la atenuación en E/LE, y también pretende demostrar cómo los estudiantes pueden mejorar sus habilidades pragmáticas relacionadas con la atenuación a través de un aprendizaje consciente que les permita desenvolverse en los diferentes contextos comunicativos, igual que los nativos.

Palabras clave: corpus oral real, atenuación, español lengua extranjera (E/LE)

\begin{abstract}
The aim of the present investigation is to point out the potentiality of using real oral discourse corpus as a didactic resource in order to teach/learn mitigation in S/SL classes. Also, we want to highlight how students can improve their pragmatic and mitigation skills, thanks to a conscious learning of those strategies that help them to communicate in different communicative contexts as well as native speakers do.
\end{abstract}

Keywords: real oral corpus, mitigation, Spanish as second language (S/SL) 


\section{Introducción}

Esta propuesta didáctica nace de una investigación doctoral (todavía en curso), en el que se ha creado un corpus oral proprio (inédito) en español, constituido por debates y conversaciones argumentativas entre estudiantes universitarios franceses aprendientes de E/LE, con un nivel de español entre un B2-C1 (según el Marco Común Europeo de Referencia para las lenguas, 2002 en adelante MCER), con el objetivo de observar y clasificar la presencia/ausencia de elementos atenuantes utilizados en las interacciones comunicativas analizadas.

A partir de esta investigación se defiende la necesidad de llevar al aula E/LE corpus discursivos reales para el análisis y aprendizaje de los mecanismos atenuantes a través de la creación y aplicación de materiales didácticos diseñados a partir de muestras extraídas de corpus orales reales.

Esta necesidad se ha demostrado en un trabajo previo (Secchi, 2014), en el que se han analizado diálogos y conversaciones empleadas en varios manuales de E/LE y cuyo resultado ha sido demostrar que en la casi totalidad de las actividades didácticas de los manuales analizados se utilizan diálogos creados ad hoc. En este mismo estudio, se han recogido unas muestras extraídas de dos corpus discursivos orales, el corpus Val.Es.Co. (2002) y el C-ORAL-ROM (2005), con el objetivo de ofrecer una visión mayor -y más realista- de los aspectos del habla cotidiana que no suelen estar presentes en los manuales de E/LE. También se destacan las ventajas de emplear corpus discursivos reales como herramientas en la didáctica de E/LE y del español como segunda lengua (E/L2), para lograr que los estudiantes adquieran las habilidades discursivas en español para que puedan desenvolverse en contextos reales de habla al igual que los nativos.

Tras este estudio, podemos afirmar que es de fundamental importancia el uso de corpus reales en el aula E/LE, sobre todo cuando los aprendientes no se encuentran en el país donde se habla la lengua meta $y$, al no encontrarse en un contexto de inmersión lingüística real, es poco probable que puedan adquirir directamente las estrategias necesarias para desenvolverse en los diferentes contextos comunicativos igual que los nativos. Es por ello que, como afirma el autor Canales (1983), es fundamental la labor del docente que actúa como puente para facilitar un aprendizaje que tenga en cuenta tanto las competencias y subcompetencias comunicativas como de los diferentes contextos comunicativos en los que se desarrollan las interacciones comunicativas.

También autores como Albelda y Fernández (2006:1), Félix-Brasdefer (2008), Holmlander (2011), entre otros, sugieren utilizar géneros textuales conversacionales reales porque presentan «un abanico muy amplio de aspectos de la lengua hablada que no suele recogerse en los materiales de E/LE y E/L2 creados ad hoc». Asimismo, afirman que se pueden diseñar actividades didácticas incluyendo interacciones orales reales a partir de los corpus transcritos existentes, que permiten enseñar al mismo tiempo tanto la lengua como la cultura. Por su parte, Briz (2002: 20) afirma que este instrumento «es la única manera de incorporar realidades no estrictamente lingüísticas al aula».

En cuanto a la inclusión de las estrategias comunicativas en el diseño de materiales en E/LE y en E/L2, como la intensificación y atenuación, también el autor Bartol (2013:52) defiende quela mejor manera de llevar al aula estas estrategias es evitar un enfoque demasiado teórico y que para lograr un mejor resultado el aprendizaje del alumno tiene que ser principalmente práctico. 
Por tanto, podemos afirmar que también otros autores consideran importante la inclusión en la enseñanza de E/LE de categoría pragmáticas como es, en el caso de nuestro estudio y propuesta, la atenuación.

\section{Punto de partida para la creación de la actividad}

El punto de partida para la creación de una actividad aplicada a la clase de E/LE ha sido plantearnos cuáles son las capacidades y las carencias del grupo de estudiantes universitarios analizado y por ello ha sido necesario plantearnos algunas preguntas de investigación, que se detallan a continuación:

- ¿Por qué enseñar la atenuación en E/LE a través de un corpus real?

- ¿Se ha estudiado cómo incorporar este fenómeno pragmático a E/LE?

- ¿Los estudiantes francófonos de E/LE conocen y son conscientes de cómo se utiliza la atenuación en la interacción oral?

- ¿Son capaces de reconocer y clasificar los elementos atenuantes en una conversación argumentativa?

- ¿Serían capaces de analizar e interpretar una posible falta de atenuación en una conversación argumentativa informal?

- ¿Bajo qué condiciones comunicativas los estudiantes acudirían a la atenuación, si tuvieran la posibilidad de modificar las interacciones en una conversación argumentativa informal?

Estas preguntas de investigación nos han permitido identificar qué elementos pretendíamos investigar y cómo podríamos hacerlo. Tras haber definido las preguntas que pretendíamos responder, hemos procedido al diseño y a la creación de una actividad didáctica a partir del análisis de un corpus discursivo real. Gracias al desarrollo de esta actividad en el aula de E/LE pretendíamos obtener unos resultados que nos indiquen, por un lado, no solamente las habilidades atenuadoras que tienen estos estudiantes, sino también cómo las aplican en función de los diferentes contextos en el que las necesitan. Por otro lado, nos permite identificar sus dificultades, sus carencias o sus faltas de conocimiento de tácticas atenuantes.

La actividad didáctica que proponemos en este trabajo ha sido creada a partir de una grabación y transcripción (según el sistema de transcripción Val.Es.Co., 2002), de una conversación argumentativa que constituye parte de un corpus oral propio (inédito) cuya temática versa sobre el aborto. Posteriormente ha sido implementada en el aula de E/LE con un grupo de estudiantes universitarios franceses de nivel B1-B2, con el objetivo de averiguar sus competencias pragmáticas relacionadas con la atenuación a través del análisis de una muestra discursiva extraída del corpus.

El desarrollo de la actividad y el posterior análisis de los resultados ha sido llevado a cabo en el laboratorio de lengua de la Université de Picardie Jules Verne (Francia), con un grupo de diez estudiantes franceses del último año de estudio en la especialidad de $L E A$ (Lenguas Extranjeras Aplicadas al comercio). Los estudiantes forman un grupo bastante homogéneo con un nivel de español B2 y todos han decidido participar de forma voluntaria al estudio.

A través de esta actividad no solo se pretende averiguar las competencias de los discentes, sino también guiarlos en el análisis de una conversación argumentativa real con el 
objetivo de identificar sus posibles carencias o falta de conocimiento de las tácticas atenuantes y sucesivamente presentarles y explicarles varias tácticas atenuadoras que faciliten un aprendizaje consciente y contextualizado de las mismas.

Con ello se pretende llevar a cabo en el aula E/LE un análisis/aprendizaje que permita a los estudiantes, guiados por el profesor, observar, analizar y adquirir, a través grabaciones y transcripciones extraídas de un corpus discursivo real, las estrategias comunicativasatenuadoras y mejorar sus destrezas productivas/receptivas que les permitirán poder desenvolverse mejor en contextos comunicativos reales al igual que los nativos.

Para el desarrollo de nuestra propuesta didáctica, en primer lugar, se ha tenido en cuenta cómo poder presentar el concepto de atenuación de la forma más resumida posible para que los estudiantes puedan familiarizarse con este concepto, y también relacionar el concepto de atenuación con la cultura en la que se aplica. Esto también les permitirá, en la fase práctica de la actividad didáctica, reflexionar acerca de la situación comunicativa que están analizando y ver las posibles diferencias culturales-comunicativas y estratégicas que podría haber entre las dos realidades lingüísticas, el francés (su lengua materna) y el español.

Los objetivos principales de nuestro trabajo han sido analizar las competencias lingüísticas atenuantes del grupo de discentes analizados y de trasladar al aula de E/LE estos conocimientos. Relacionado con estos objetivos, nos hemos planteado otros objetivos específicos que nos permitan averiguar lo siguiente:

- Si los discentes son capaces de reconocer los diferentes elementos atenuantes presentes en la grabación/transcripción

- Si saben reconocer las diferentes funciones de las tácticas atenuantes presentes en la transcripción analizada

- Si son capaces de proponer tácticas atenuadoras alternativas a la que hayan encontrado en la transcripción analizada

- Si saben identificar una posible carencia de atenuación y proponer una posible solución adecuada al contexto comunicativo analizado

Tras un primer análisis, guiado por el profesor-investigador, los estudiantes identificarán la presencia/ausencia de elementos atenuadores, sus funciones y su adecuada utilización en función del contexto.

Esta reflexión y puesta en común es el punto de partida para la puesta en práctica de la actividad didáctica. Los estudiantes tienen que identificar si en la grabación/transcripción de la conversación argumentativa hay elementos atenuadores $\mathrm{y}$, en el caso de que para ellos no los hubiese, decidir cuáles serían entre los elementos atenuadores que conocen los que se podrían incluir en las interacciones.

En la fase siguiente, el profesor les enseña una lista de elementos atenuadores y sus funciones. A partir de ahí, los discentes vuelven a analizar la transcripción de la conversación argumentativa y deciden si, con los nuevos elementos que tienen, sería oportuno incluir en las interacciones algunos elementos atenuadores que anteriormente no habían considerado.

Para la creación de nuestra actividad didáctica hemos decidido utilizar una grabación oral informal-y su transcripción- extraída de un corpus oral proprio, cuya temática versa sobre el aborto. A través del análisis de la muestra, se pretende que los discentes activen sus 
conocimientos previos en cuanto a las funciones comunicativas. Para ello, tendrán que formular hipótesis sobre el contenido léxico-pragmático y atenuante partiendo del análisis de la interacción oral. Gracias a ello, se pretende que amplíen sus conocimientos en cuanto a los elementos atenuantes relacionados con la conversación argumentativa, reflexionen sobre las características que pueden diferenciar los registros lingüísticos (formal, informal, semiformal), y por último que identifiquen los elementos atenuantes presentes en la transcripción analizada de modo que posteriormente puedan proponer posibles cambios donde consideren que haya una carencia de atenuación.

\section{Descripción de la muestra llevada al aula E/LE}

La grabación de la conversación argumentativa tiene una duración de 4 minutos y 57 segundos en la que participan dos mujeres (etiquetadas como A y B), de edad entre $20 \mathrm{y}$ 25 . El tema de la conversación argumentativa gira en torno al aborto. Este tema ha sido elegido expresamente porque es una buena muestra extraída del corpus en la que se puede apreciarla posición a favor del aborto por parte del hablante A y la posición en contra del aborto por parte del hablante B. Además, en esta conversación argumentativa no solo encontramos varios elementos y tácticas atenuantes, sino también algunas intervenciones que podrían haber sido atenuadas y no lo han sido y que por lo tanto representan un ataque al imagen del interlocutor. Estas carencias atenuantes servirán en una de las fases de la actividad didáctica, en las que los discentes tendrán que proponer -según su opiniónqué tácticas o elementos atenuantes podrían haber utilizado los dos hablantes (A y B) en algunos de los intercambios que constituyen un ataque a la imagen propia o del interlocutor y que hubieran podido ser atenuadas.

\section{Desarrollo de la actividad didáctica}

Para la creación de nuestra actividad didáctica hemos decidido utilizar una grabación y una transcripción informal extraída de un corpus oral propio (inédito), cuya temática versa sobre el aborto.

A continuación, se detallan los pasos que hemos dado para la puesta en práctica de la actividad didáctica llevada a la clase de E/LE.

\subsection{Consignas y fases de la actividad}

Se ha avisado a los estudiantes de que se les entregarán varios documentos en las diferentes etapas de la actividad y que tendrán que seguir las indicaciones que les dará el profesor. Se les ha entregado el primer documento con la transcripción de un audio, se les ha informado de que primero van a leer la transcripción, y que junto a la transcripción aparecen varios signos de transcripción (Briz y grupo Val.Es.Co. 2002), que se les explicarán a lo largo de la actividad. Posteriormente se les han detallado los pasos que tendrán que seguir: primero escucharán un audio -dos veces-, después leerán la transcripción del audio - dos veces- y por último, escucharán el audio - una vez más-y leerán simultáneamente la transcripción. También se les ha informado de que el género discursivo de la grabación es una conversación argumentativa informal, cuya temática versa sobre el aborto y que sus participantes son dos mujeres de edad entre 20 y 25 años. Estas indicaciones les serán útiles para otra fase de la actividad didáctica en las que 
tendrán que buscar elementos atenuantes e proponer posibles cambios teniendo en cuenta de contexto comunicativo.

Se les ha preguntado si sabían qué es la pragmática y si entre todos -tras una puesta en común- eran capaces de dar una pequeña definición en español. Esta actividad no solo ha servido para animar el grupo a formular una hipótesis, sino también para introducir otra parte de la actividad en las que se les explicarán algunos conceptos base útiles posteriormente para poder avanzar con la actividad.

Una vez terminada esta parte de actividad, se ha dado inicio a las cuatro fases de análisis (Análisis 1; Análisis 2, Análisis 3; Análisis 4) alternadas de algunas explicaciones teórico-prácticas por parte del profesor, y que se detallan a continuación.

\subsubsection{Análisis 1: Identificación de la presencia de atenuación por parte de los estudiantes (antes de entregar dos definiciones de atenuación).}

Tras la puesta en común, se les ha pedido que volviesen a leer la transcripción de la grabación y que, trabajando individualmente, subrayaran cuáles elementos, según ellos, constituían un ataque al interlocutor A y/o B y que justificaran su elección. Se pueden ver los elementos seleccionados por los estudiantes como ataque (daño a la imagen del interlocutor o del yo del propio hablante) en la transcripción de la grabación (apartado 5), donde han sido marcados de color amarillo. Para el desarrollo de esta parte de actividad se había previsto un máximo de 15 minutos. Posteriormente, se les ha entregado el segundo documento en el que se incluyen una definición de competencia pragmática seguida de algunos ejemplos- y dos definiciones de atenuación: la primera es una definición del Diccionario de Lingüística Moderna (2004) y la segunda una definición operativa del concepto de atenuación (Albelda y Briz, 2010: 238; 2013).

Estas tres definiciones permiten que los estudiantes tengan un apoyo teórico de partida que les permita reflexionar sobre la función de la pragmática y de la atenuación en los intercambios comunicativos contextualizados en español.

A continuación se les ha explicado a los estudiantes el por qué se atenúa (suavizar el mensaje, quitar relieve, mitigar, reparar, esconder la verdadera intención del hablante) dándoles varios ejemplos. Para definir estos valores nos hemos apoyado en la clasificación de Briz y Albelda (2013:302) que distinguen tres funciones atenuantes: autoprotección, prevención y reparación.

\subsubsection{Análisis 2: Identificación de la presencia de atenuación por parte de los estudiantes (después de entregar dos definiciones de atenuación).}

Una vez definida -y explicada- la parte teórica hemos procedido con otra fase de la actividad, en la que se ha pedido a los estudiantes que volviesen al primer documento de la transcripción de la grabación, que volviesen a leerla y que intentaran identificar cuáles eran según ellos las tácticas o elementos atenuantes utilizados por los participantes A y B en la conversación argumentativa y que justificaran su elección. Se pueden ver los elementos seleccionados por los estudiantes como atenuantes en la transcripción de la grabación (apartado 5), donde han sido marcados de color azul. Para el desarrollo de esta parte de actividad se había previsto un máximo de 15 minutos. 


\subsubsection{Análisis 3: Identificación de la presencia de elementos de ataque a la imagen por parte de los estudiantes (antes de explicar las funciones $y$ variables atenuadoras).}

Tras la puesta en común, se les ha pedido que volviesen a leer la transcripción de la grabación y que -siempre trabajando individualmente- subrayaran nuevos elementos (que no habían sido seleccionados anteriormente) y que para ellos constituían un ataque para el participante A y/o B. Se pueden ver los elementos seleccionados por los estudiantes como ataque (daño a la imagen del interlocutor o del yo del propio hablante) en la transcripción de la grabación (apartado 5), donde han sido marcados de color fucsia. Posteriormente se ha pedido a los estudiantes que substituyeran los elementos de ataque con otros elementos atenuantes que ellos consideraban oportunos, ateniéndose al contexto comunicativo. Para el desarrollo de esta parte de actividad se había previsto un máximo de 15-20 minutos.

A continuación, se les ha entregado el tercer documento, en la que se enumeran dieciséis tácticas lingüísticas y no lingüísticas de atenuación (extraídas de la ficha metodológica para el análisis de la atenuación en corpus discursivos del español, Albelda et alii, 2014 pp. 7-62). A continuación incluimos el contenido del tercer documento que ha sido entregado a los estudiantes:

Tácticas lingüísticas y no lingüísticas de atenuación

1. Modificadores morfológicos internos: sufijos, diminutivos

2. Modificadores externos: cuantificadores minimizadores, aproximativos o difusores significativos proposicionales o extraproposicionales

3. Empleo de palabras extranjeras

4. Verbos, construcciones verbales y partículas discursivas modales que expresan opinión en forma de duda o de probabilidad

5. Verbos, construcciones verbales y partículas discursivas que expresan fingimiento de incertidumbre, de incompetencia o de ignorancia

6. Construcciones acotadoras de la opinión a la propia persona o a un determinado ámbito

7. Peticiones, preguntas, mandatos u órdenes expresadas de forma indirecta

8. Expresiones de disculpa

9. Construcciones justificadoras o de excusa

10. Impersonalizaciones

11. Movimiento de reformulación

12. Concesividad

13. Partículas discursivas modales de objetivación

14. Partículas discursivas y expresiones de control de contacto con el interlocutor

15. Elementos prosódicos, paralingüísticos o gestuales: risas; alargamiento vocálico; subido o bajada de tono

Por cada táctica se han dado algunos ejemplos prácticos y se les ha entregado a los estudiantes otros cuatro documentos con los relativos ejemplos que no incluimos en este trabajo por problemas de extensión del artículo. 


\subsubsection{Análisis 4: Identificación de la presencia de atenuación por parte de los estudiantes (tras la explicación por parte del investigador de algunas funciones $y$ variables atenuadoras).}

Tras la puesta en común, se les ha pedido que volviesen al documento de la transcripción de la grabación y -siempre trabajando individualmente- subrayaran nuevos elementos no seleccionados anteriormente- que para ellos representaban tácticas o elementos atenuantes.

En esta fase los estudiantes han sido guiados por el profesor que les ha ayudado en el proceso de identificación de los elementos atenuantes a través de la lista de clasificación de las tácticas atenuantes que le ha sido entregada. Con ello se pretende que los discentes familiaricen con los elementos atenuantes que están utilizando y que aprendan -de forma consciente- cuáles son los resultados que pueden conseguir al usar unos elementos atenuantes más que otros y qué función cumple cada elemento. De esta forma se favorece un aprendizaje consciente puesto que los estudiantes se entrenarán en elegir qué elementos atenuadores quieren utilizar en sus interacciones orales teniendo en cuenta los objetivos que quieren conseguir.

Se pueden ver los elementos seleccionados por los estudiantes como atenuantes en la transcripción de la grabación (apartado 5), donde han sido marcados de color verde. Para el desarrollo de esta parte de actividad se había previsto un máximo de 15 minutos.

\section{Transcripción de la grabación de la conversación argumentativa informal}

Número y sexo de los participantes: 2 mujeres - Edad de los participantes: $<25$ años

Temática: el aborto.

Los colores tienen la función de indicar los diferentes elementos seleccionados por parte de los estudiantes, en cada una de los cuatro análisis, y que se indican a continuación.

Se indican en amarillo los elementos que para los estudiantes constituyen un ataque a la imagen de A y/o B (Análisis 1), en azul los elementos atenuantes (Análisis 2), en fucsia los elementos de ataque a la imagen del interlocutor A y/o B (Análisis 3), y en verde los elementos atenuantes (Análisis 4):

1 A: bueno emm/ sobre el aborto tú piensas que es una buena idea autorizar el aborto

2 en Francia// por ejemplo

3 B: bueno/ yo pienso que puede ser una buena idea// pero depende las condiciones//

4 emm por ejemplo yo pienso que podríaa ser legalizados peroo/ en caso en quee $\rightarrow$ la

5 madre/// bueno huígan de la madre/ estaría en peligro peroo $\rightarrow$ del resto yo pienso

6 que hay que darle la vida al niño// que pase lo que pase porque es algoo $\rightarrow$ bueno es

7 algo $\rightarrow$ natural / y es algo que paso/ y eso no see $\rightarrow / /$ o sea por ley y por/ emm- por-

8 por amor/ tú no puedes dejar a un niño puees/morir

9 A: entiendo lo que dices peroo no estoy de acuerdo contigo $1 / /$ para Míl/ la mujer

10 tienes que tener/ tiene quee poder elegir entre las dos opciones/ no sabemos lo que ha

11 pasado antes/ no sabemos si el- bueno el paadre está presente o no está presente/ a lo

12 mejor/// emm bueno/ no- no sabemos el pasado de la mujer/ no podemos decir para

13 ella que guarde el bebé sin- sin saber lo que pasó $\S$

14 B:

15 que pagar por los errores sus papas/o sea/ no es porque tus papas hicieron un error/

16 que tú tienes que pagar tu vida por eso $\S$

\footnotetext{
${ }^{1}$ Entre risas.
} 
18 pasa? y no tiene familia/ noo- su familia no está- no noo hombre/ o no está feliz/ no-

19 no tiene padres/su madre no puede educarlo/ no puedee/ a lo mejor es- bueno a lo

20 mejor sería/// una buena ideaa quee $\rightarrow /$ ¡no! yo no ${ }^{2}$ - yo pienso que es una bue- muy

21 mala idea/ [y]

22 B: [ya] peroo yo pienso que siempre se encuentran soluciones/ aunquee

23 hay muchos niños que nacen sin- sin padres siempre/ hay familias que puede ayudar/

24 siempree hayy establecimientos que pueden también ayudaar/ la religión católica que

25 puede también ayudar muchíisimo/ emm yo pienso que un niño/ cuando nace nunca

26 está solo yy $\rightarrow$ sí pue- hay niños que no tengo padres/ pero eso no quieres decir que

27 más tarde su vidaa $\rightarrow$ estaráa/ [o seaa $\rightarrow$ ]

28 A: [i- imaginate] ahora tienes buenoo/ como dieciocho

29 diecinueve años bueno/ yy y tienes un niño ahora ¿qué haces? ¿lo vas- lo vas a

30 guardar? estás haciendo tus estudios/ te vas a ir a México// no- no puedes seguir/

31 ¿vas a- vas a parar tu vida ahora?/ bueno// entiendo lo que dices peroo ¿vas parar tu $32 \mathrm{vida/}$ tus estudios// ahora?/// emm $\S$ familia yoo pararía todo// aunque sea un niño oo una mamá o un papá/ yo creo queeque son/sacrificios que se hacen y que más tarde pues/ nos pueden beneficiar a todos/ así quee// yo sin duda// yo pararía too hasta quee/ y yo pienso de todas manera quee yo sería feliz si tengo un niño/// ahora mismo

A: ¿tú no piensas que/ sería una buena idea emm/ dejar- bueno/// yo se que en Francia conozco- buenoo/ tuve un amiga que tuvo que abortar/ ee cono- yo se que en Francia tienes una semana de reflexión// puedes hablar con un médico/ TIENES que hablar con un médico/ que te autoriza y te dice bueno/ SÍ puedes/ si tienes las razones/// eso también// puede ser una idea para que no todas las chicas aborten así sin pensar $§$

B: $\quad \S$ totalmente de acuerdo con eso/ eso es super importante que esté eflexionado antes/ peroo $\rightarrow$ / aunque esté legalizado yoo- realmente yo creo quee $\rightarrow$ que entre la vida y entre la muertee $\rightarrow$ / escoger la vidaa $\rightarrow$ / es algo natural y es algo primordial/// yoo $\rightarrow$ no sé co/// tú por ejemplo/ ¿a ti no te daría mucho (( )) saber que tú mataste tu hijo?

A: si tiene que pasar (3') yoo- [buenoo a lo mejor]
B:
[sí pero imagínate que] toda tu vida tu tendrás en la conciencia un hijo que tu ya harás podido tener $\S$

A: $\S$ peroo ¡si no tengo otra opción! yoyo no me veo ahoraa tener un hijo coon diecinueve años// sii $\rightarrow$ tengo que- tengo quee $\rightarrow$ estudiar todavía/// noo $\rightarrow$ para mí no- no tengo dinero ¿qué voy a hacer?/// [eso no es un]

B: [como te lo dije]// si tienes familia lo puedes cuidar $\S$

A:

$\S$ claro peroo no es laa $\rightarrow$ no

es la vida que espero para miss hijos $\S$

B:

A:

$\S$ [yaa]

$[(())]($ RISAS)

\section{Resultados parciales}

En este apartado se presentan los resultados más relevantes de los cuatro análisis de la transcripción de la conversación argumentativa llevadas a cabo por los estudiantes.

\subsection{Resultados del Análisis 1. Elementos identificados (en amarillo)}

tú no puedes dejar a un niño puees/ morir (línea 8); no estoy de acuerdo contigo // (línea 9); yo pienso que es una bue- muy mala idea/ (línea 20-21) tú mataste tu hijo? (línea 48).

\footnotetext{
${ }^{2}$ Entre risas.
} 
Total de los elementos identificados: 4 y total de los diferentes elementos identificados: 4.

\subsection{Resultados del Análisis 2. Elementos identificados (en azul)}

yo pienso (línea 3, 5, 22, 25, 36); entiendo lo que dices peroo (línea 9); bueno (línea 17); a lo mejor (línea 19); ya pero (línea 22); entiendo lo que dices peroo(línea 31) puede ser (línea 33); ¿tú no piensas que/ sería una buena idea (línea 38); eso también (línea 42); puede ser una idea (línea 42); totalmente de acuerdo con eso (línea 35-36) buenoo a lo mejor (línea 49); ya te entiendo/// pero// (línea 61). Total de los elementos identificados: $17 \mathrm{y}$ total de los diferentes elementos identificados: 13.

\subsection{Resultados del Análisis 3. Elementos identificados (en fucsia)}

para MÍl (línea 9); ya peroo el niño no vaa- no tiene que pagar por los errores sus papas (línea 14-15); no es porque tus papas hicieron un error/ que tú tienes que pagar tu vida por eso (línea15-16); y sii- y si el niño nace/ yy- y ¿qué pasa? (línea 17-18) ¡no! (línea 20); ¿qué haces? ¿lo vas- lo vas a guardar? (línea 29-30); ¿vas a- vas a parar tu vida ahora? (línea 31); ¿vas a parar tu vida/ tus estudios// ahora? (línea 31-32) TIENES que hablar con un médico (línea 40-41); Sí puedes (línea 41); no todas las chicas aborten así sin pensar (línea 42-43); entre la vida y entre la muertee $\rightarrow$ / escoger la vidaa $\rightarrow$ / es algo natural y es algo primordial (línea 46-47); toda tu vida tu tendrás en la conciencia un hijo que tu ya harás podido tener (línea 50-51); isi no tengo otra opción! (línea 52); como te lo dije (línea 56). Totalidad de los elementos identificados: 15 y totalidad de los diferentes elementos identificados: 15.

\subsection{Resultados del Análisis 4. Elementos identificados (en verde)}

puede ser una buena idea// pero (línea 3 ); yo pienso que podríaa ser legalizados peroo (línea 4); no see $\rightarrow / /$ (línea 7); a lo mejor (línea 11-12); i- imagínate (línea 28) ; yo creo (línea 34); eso es super importante que esté reflexionado antes/ pero (línea 44-45) yoorealmente yo creo quee $\rightarrow$ (línea 45); yoo $\rightarrow$ no sé (línea 47); sí pero (línea 50) para mí (línea 54); claro pero (línea 57); yaa (línea 59); RISAS (línea 60, 62); no sé (línea 61). Totalidad de los elementos identificados: 16 y totalidad de los diferentes elementos identificados: 15

\section{Interpretación de los datos obtenidos en cuanto al uso}

Los estudiantes saben reconocer y utilizar algunas de las tácticas de atenuación para la salvaguarda del yo-tú (imagen y protección de la imagen), de prevención y reducción de conflictos, de desacuerdo y de rechazo a pesar de que la función del atenuante más frecuente que han utilizado en sus cambios ha sido la de prevención. Por lo tanto, el análisis del juicio pragmático sobre el uso del atenuante nos indica que los informantes conocen y saben utilizar algunas de las tácticas y elementos atenuadores pero que sus habilidades pragmáticas en contexto son limitadas.

\section{Conclusiones}

Con este trabajo hemos querido por un lado, averiguar el conocimiento de las habilidades atenuadoras de un grupo de estudiantes universitarios franceses, gracias al uso de un corpus discursivo real (oral y escrito) en el aula de E/LE. Por otro lado, se ha querido demostrar la rentabilidad de este instrumento como herramienta de aprendizaje de tácticas y elementos atenuantes que pueden permitir un aprendizaje consciente y más eficaz de 
las habilidades pragmáticas relacionadas con la atenuación. Esta actividad ha tenido como objetivo demostrar que el binomio del uso de corpus orales reales y la enseñanza de la atenuación en E/LE representa un medio imprescindible para que los estudiantes aprendan a desenvolverse en diferentes contextos de habla al igual que los nativos.

\section{Bibliografía}

AlBELDA M., \& M. J. FERNÁNDEZ (2006). La enseñanza de los registros lingüísticos en E/LE. Una aplicación a la conversación coloquial. Recuperado el 14/3/17 de: ww.marcoele.com/num/3/0218f5989b0f06708/coloquial.pdf.

Albelda, M., A., Cestero, A., KotwicA, D., \& Villalba, C. (2014). «Ficha metodológica para el análisis pragmático de la atenuación en corpus discursivos del español (ES. POR. ATENUACIÓN)». Oralia, 17, pp. 7-62.

AlBeldA, M., \& A. BRIz (2010). «Aspectos pragmáticos. Cortesía y atenuantes verbales en las dos orillas a través de muestras orales». En M. Aleza \& J. Enguita (coords.). La lengua española en América: Normas y usos actuales (pp. 237-260). Valencia: Universitat de València.

Alcaraz, E., V. \& Martínez Linares, M.A. (2004). Diccionario de lingüistica moderna. Barcelona: Ariel.

BARTOL, E. (2013). «La atenuación y la intensificación: herramientas para un mensaje adecuado». Tinkuy: Boletín de investigación y debate, 19, pp. 47-55.

BRIZ, A. \& AlBELDA, M. (2013). «Una propuesta teórica y metodológica para el análisis de la atenuación lingüística en español y portugués: La base de un proyecto en común (ES.POR.ATENUACIÓN)». Onomázein: Revista de lingüística, filología y traducción de la Pontificia Universidad Católica de Chile, 28, pp. 288-319.

BRIZ, A. \& grupo Val.Es.Co. (2002). Corpus de conversaciones coloquiales. Madrid, Arco Libros (Anejos de la revista Oralia).

BRIz, A. (2002). El español coloquial en la clase de E/LE: un recorrido a través de los textos. SGEL: Sociedad General Española de Librería.

CANALE, M. (1995). «De la competencia comunicativa a la pedagogía comunicativa del lenguaje». Llobera (coord.). Competencia comunicativa (pp. 63-81). Madrid: Edelsa.

CONSEJO de Europa (2002). Marco Común Europeo de Referencia para el aprendizaje, la enseñanza y la evaluación de lenguas. Madrid: Instituto Cervantes, MECD, Anaya.

CRESTI, E. \& Moneglia, M. (Eds.). (2005). C-ORAL-ROM: integrated reference corpora for spoken romance languages (Vol. 15). John Benjamins Publishing.

FÉLIX-BRASDEFER, J. (2008). «Teaching pragmatics in the classroom: Instruction of mitigation in Spanish as a Foreign Language». Hispania, 89, pp. 479-494.

Holmlander, D. (2011). Estrategias de atenuación en español L1 y L2: estudio contrastivo en hablantes españoles y suecos (Vol. 89). Lunds universitet.

SECCHI, D. (2014). De la comunicación oral dirigida a la autonomía comunicativa: la enseñanza de E/LE a través de corpus discursivos. Recuperado el 20/01/17, de: http://www.mecd.gob.es/redele/Biblioteca-Virtual/2014/memorias-master/Daniel-

Secchi.html 